\title{
NOTES ON THE FISHES COLLECTED BY THE R.V. "TRIDENS" ON THE NORTH WEST AFRICAN SHELF, 19-25 JANUARY 1969
}

\author{
by \\ S. J. DE GROOT \& H. NIJSSEN \\ Netherlands Institute for Fishery Investigations, IJmuiden, The Netherlands \\ and \\ Institute of Taxonomic Zoology (Zoölogisch Museum), University of Amsterdam, The Netherlands
}

\begin{abstract}
On the North West African shelf 98 species of fishes were collected of which a classification is given. Three species have hitherto been recorded only farther South. The status of Platophrys podas africanus (Nielsen, 1961) is discussed.
\end{abstract}

\section{INTRODUCTION}

During a voyage of the Dutch fishery research vessel "Tridens", of the Ministery of Agriculture and Fisheries, fishes were collected in the waters of the North West African shelf from Cape Bojador to Cape Blanc in the period 19-25 January 1969. Initially the voyage was intended to study the use of different types of pelagic fishing gear in deep waters, deprived of any fish if possible, as they would disturb the technical mearurements. However, at the time our ship was due to sail, Dutch trawler owners asked the Ministery for information about the fishery possibilities of Dutch vessels in North West African waters for the months January and February, when in European waters fishery possibilities are rather poor. The area in which the technical experiments would be carried out being the waters around the Canary Islands, it was decided to extend the voyage by a week to obtain an impression of the fauna of this area at that time. We thought it justifiable to report here upon the results of our short survey, because data on the fish and fisheries in waters of northwestern Africa are rather scarce. In addition to data on the composition of the trawl catches at the different stations (fig. 1), the classification of the 98 species collected (specimens of which are deposited at the Institute of Taxonomic Zoology, Zoölogisch Museum, Amsterdam) is provided. Station numbers 5 to 9 refer to the Arguin Bank. At stations 1 and 11 a pelagic trawl, at stations 2 to 10 a herring trawl was used. The time given is local time.

\section{LIST OF STATIONS (fig. 1)}

Station 1: 20-I-1969; time 14.20-16.15 h.; pelagic trawl; depth $94-100$ fms.; $25^{\circ} 43^{\prime}-26^{\circ} 34^{\prime} \mathrm{N}$, $15^{\circ} 21^{\prime}-15^{\circ} 22^{\prime} \mathrm{W}$.

Remarks. - Two specimens of the Scabbard Fish (Lepidopus caudatus) and one John Dory (Zeus faber) and about $30 \mathrm{~kg}$ of the pelagic ascidian Pyrosoma atlanticum were caught.

Station 2: 20-I-1969; time $20.20-21.00 \mathrm{~h}$; herring trawl; depth $34-35 \mathrm{fms}$; $25^{\circ} 01^{\prime}-24^{\circ} 59^{\prime} \mathrm{N}, 16^{\circ} 25^{\prime}$ $-16^{\circ} 28^{\prime} \mathrm{W}$.

Remarks. - We fished in the centre of a small fleet of Spanish cutters to investigate the species they were fishing for; these proved to be mainly cephalopods. Total catch about $100 \mathrm{~kg}$ of which $30 \mathrm{~kg}$ Loligo species, $30 \mathrm{~kg}$ Sepia species, $10 \mathrm{~kg}$ Octopus species, $15 \mathrm{~kg}$ Weevers (Trachinus), 10 $\mathrm{kg}$ flatfish, mainly Soles (Dicologoglossa azevia and D. cuneata), $5 \mathrm{~kg}$ sea perches (Dentex, Spondyliosoma).

Station 3: 21-I-1969; time 09.30-10.30 h.; herring trawl; depth $46-49 \mathrm{fms}$.; $23^{\circ} 09^{\prime}-23^{\circ} 06^{\prime} \mathrm{N}, 17^{\circ} 03^{\prime}$ $17^{\circ} 02^{\prime} \mathrm{W}$.

Remarks. - Total catch about $300 \mathrm{~kg}$ of which $160 \mathrm{~kg}$ Horse Mackerel (Trachurus trachurus), $35 \mathrm{~kg}$ small Gurnards (Aspitrigla obscura), $30 \mathrm{~kg}$ sea perches, $20 \mathrm{~kg}$ Spanish Mackerel (Scomber japonicus), $15 \mathrm{~kg}$ sharks and rays, $15 \mathrm{~kg}$ Stargazers (Uranoscopus), $5 \mathrm{~kg}$ flatfish, $15 \mathrm{~kg}$ others.

Station 4: 21-I-1969; time 14.10-15.30 h.; herring trawl; depth $47-50$ fms.; $22^{\circ} 23^{\prime}-22^{\circ} 15^{\prime} \mathrm{N}, 17^{\circ} 15^{\prime}$ $-17^{\circ} 14^{\prime} \mathrm{W}$. 


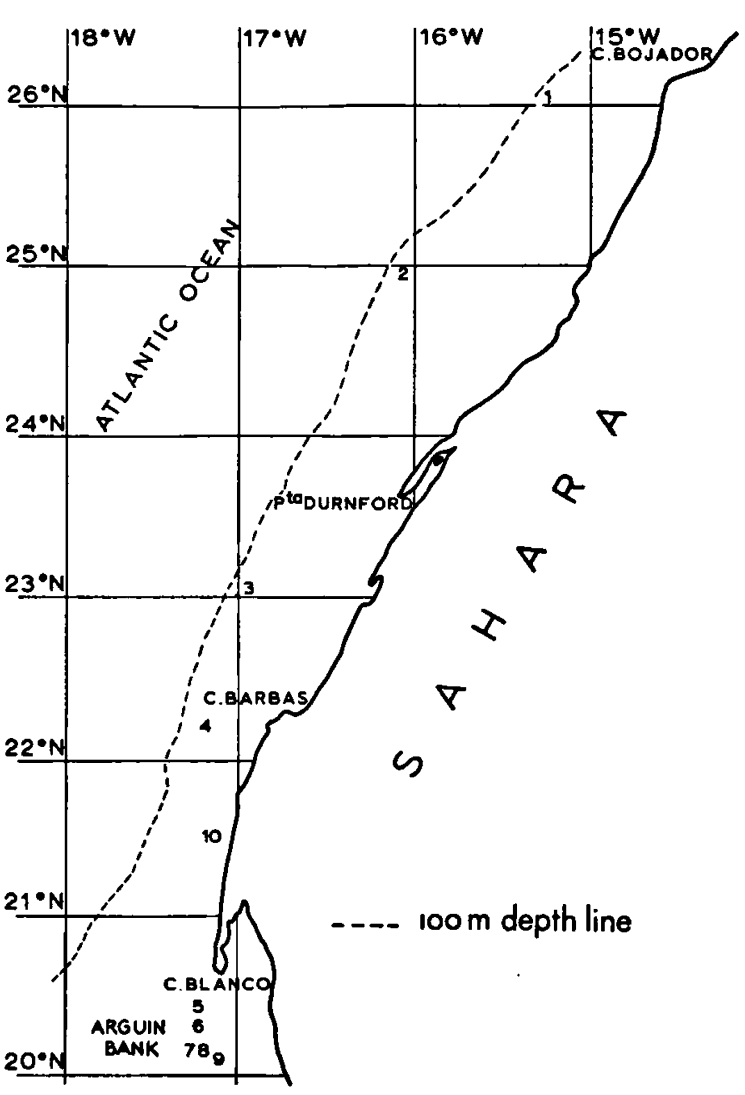

Fig. 1. Map of North West African shelf with position of stations 1 to 10 .

Remarks. - We fished along trawling Russian and Bulgarian vessels which were mainly fishing for Horse Mackerel as our trawlcatch showed. Total catch about $1000 \mathrm{~kg}$ of which $800 \mathrm{~kg}$ Horse Mackerel, $100 \mathrm{~kg}$ sea perches, $20 \mathrm{~kg}$ Gurnards, $20 \mathrm{~kg}$ Stargazers, $20 \mathrm{~kg}$ Octopus, $5 \mathrm{~kg}$ Loligo, $15 \mathrm{~kg}$ flatfish of which $10 \mathrm{~kg}$ large Soles, $10 \mathrm{~kg}$ Spanish Mackerel.

Station 5: 21-I-1969; time 07.15-08.15 h.; herring trawl; depth $22-24$ fms.; $20^{\circ} 35^{\prime}-20^{\circ} 37^{\prime} \mathrm{N}$, $17^{\circ} 24^{\prime}-17^{\circ} 22^{\prime} \mathrm{W}$.

Remarks. - Total catch about $1100 \mathrm{~kg}$ of which $900 \mathrm{~kg}$ sea perches $(700 \mathrm{~kg}$ Plectorhynchus mediterraneus, $200 \mathrm{~kg}$ Epinephelus aeneus), $80 \mathrm{~kg}$ large carangids (Caesiomorus vadigo, Decapterus punctatus), $90 \mathrm{~kg}$ sciaenids (60 kg Atractoscion aequidens, $30 \mathrm{~kg}$ Umbrina), $50 \mathrm{~kg}$ rays, $40 \mathrm{~kg}$ Gurnards, $30 \mathrm{~kg}$ Congereels, $10 \mathrm{~kg}$ flatfish, $40 \mathrm{~kg}$ others.
Station 6. 22-I-1969; time 08.45-11.15 h.; herring trawl; depth $24-25 \mathrm{fms}$.; $20^{\circ} 36^{\prime}-20^{\circ} 18^{\prime} \mathrm{N}, 17^{\circ}$ $22^{\prime}-17^{\circ} 27^{\prime} \mathrm{W}$.

Remarks. - Total catch about $1600 \mathrm{~kg}$, of which $1200 \mathrm{~kg}$ sea perches, $150 \mathrm{~kg}$ Anchovy, $100 \mathrm{~kg}$ carangids (Decapterus), $40 \mathrm{~kg}$ sciaenids, $40 \mathrm{~kg}$ cephalopods, $30 \mathrm{~kg}$ Hairtail (Trichiurus), $5 \mathrm{~kg}$ flatfish (Soles), $40 \mathrm{~kg}$ others.

Station 7: 22-I-1969; time 15.00-15.45 h.; herring trawl; depth 9-11 fms.; $20^{\circ} 12^{\prime}-20^{\circ} 07^{\prime} \mathrm{N}, 17^{\circ} 15^{\prime}$ $-17^{\circ} 18^{\prime} \mathrm{W}$.

Remarks. - Total catch about $750 \mathrm{~kg}$ of which $450 \mathrm{~kg}$ sea perches (Dentex) $100 \mathrm{~kg}$ Anchovy, 50 $\mathrm{kg}$ sea Catfish (Arius), $40 \mathrm{~kg}$ sciaenids, $30 \mathrm{~kg}$ cephalopods, $30 \mathrm{~kg}$ sharks, $30 \mathrm{~kg}$ rays, $30 \mathrm{~kg}$ Hairtail, $30 \mathrm{~kg}$ others.

Station 8: 22-I-1969; time 20.00-21.15 h.; herring trawl; depth 10 fms.; $20^{\circ} 10^{\circ}-20^{\circ} 14^{\prime} \mathrm{N}, 17^{\circ} 12^{\prime}-$ $17^{\circ} 10^{\circ} \mathrm{W}$.

Remarks. - We fished along a fleet of thirteen large Japanese trawlers, who were presumably fishing for shrimp (Penaeus) and sea perches. Total catch about $200 \mathrm{~kg}$ of which $100 \mathrm{~kg}$ sea perches, $50 \mathrm{~kg}$ sciaenids, $50 \mathrm{~kg}$ others. Among the crustaceans we found three forms of Penaeidae, Penaeus kurathurus (Forskål), Penaeus duorarum Perez Farfante, and Metapenaeopsis miersi (Holthuis).

Station 9: 22-I-1969; time 22.30-23.15 h.; herring trawl; depth $10 \mathrm{fms}$; $20^{\circ} 11^{\prime}-20^{\circ} 05^{\prime} \mathrm{N}, 17^{\circ} 13^{\prime}-$ $17^{\circ} 15^{\prime} \mathrm{W}$.

Remarks. - Net was damaged, but still we caught Horse Mackerel, sea perches, and Stromateus.

Station 10: 23-I-1969; time 09.30-11.00 h.; herring trawl; $40-58$ fms.; $21^{\circ} 35^{\prime}-21^{\circ} 58^{\prime} \mathrm{N}, 17^{\circ} 22^{\prime}-$ $17^{\circ} 19^{\prime} \mathrm{W}$.

Remarks. - Total catch about $700 \mathrm{~kg}$ of which $400 \mathrm{~kg}$ Horse Mackerel, $150 \mathrm{~kg}$ sea perches (Dentex, Spondyliosoma), $50 \mathrm{~kg}$ Spanish Mackerel, 80 $\mathrm{kg}$ other fishes (Stargazer, Angler fish).

Station 11: 25-I-1969; time 14.20-14.35 h.; pelagic trawl; depth $350 \mathrm{fms}$.; $27^{\circ} 48^{\prime} \mathrm{N}, 15^{\circ} 12^{\prime} \mathrm{W}$.

Remarks. - About 20 species of salmonid fishes (myctophids, Hatched Fishes and others). 

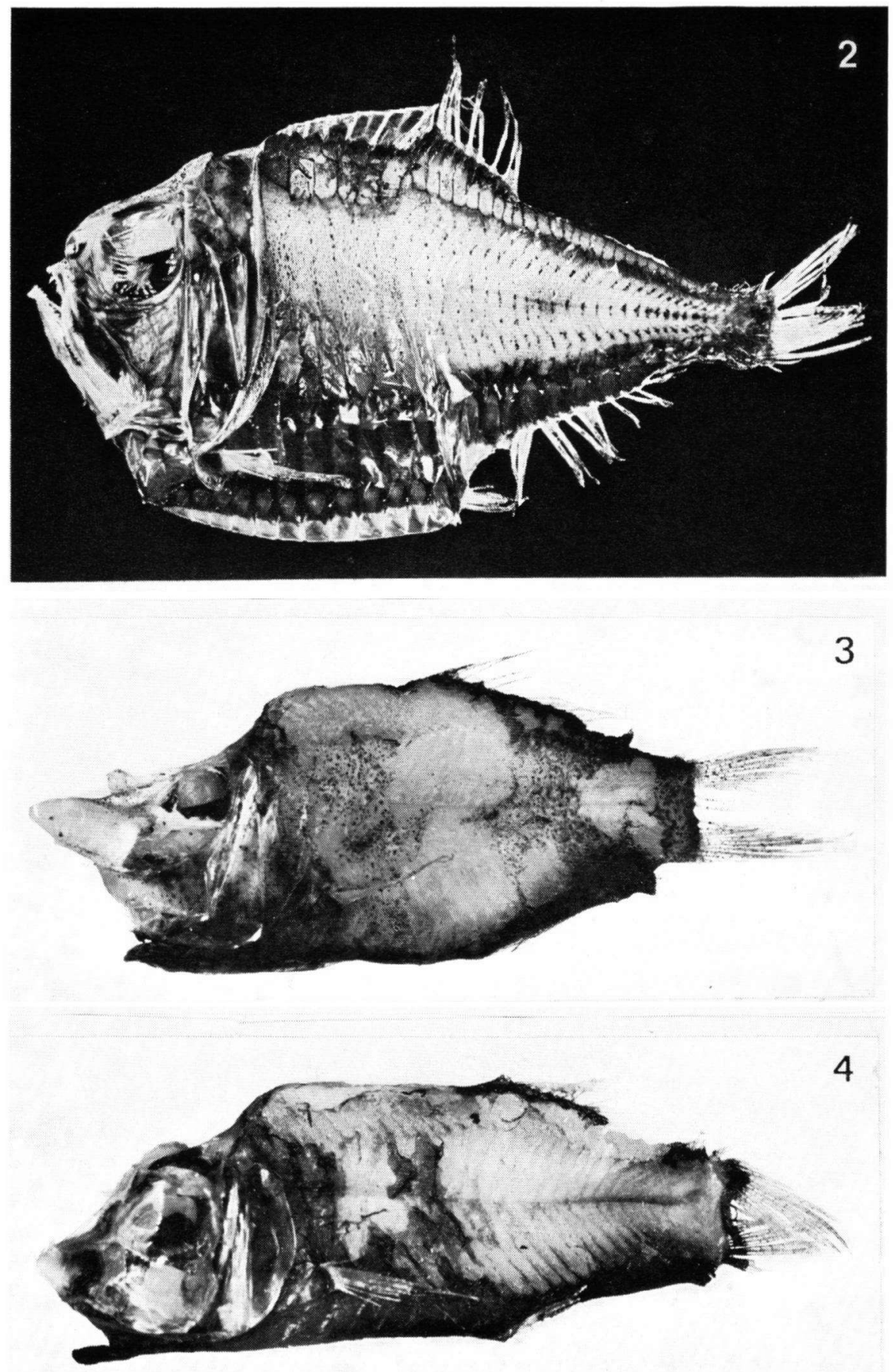

Fig. 2. Argyropelecus gigas Norman, 1930, $87.3 \mathrm{~mm}$ sl, from station 11 (ZMA 109.920).

Fig. 3. Opisthoproctus grimaldii Zugmayer, 1911, $50.1 \mathrm{~mm}$ sl, from station 11 (ZMA 109.935).

Fig. 4. Opisthoproctus soleatus Vaillant, 1888, $50.6 \mathrm{~mm}$ sl, from station 11 (ZMA 109.936). 


\section{CLASSIFICATION OF THE FISHES COLLECTED}

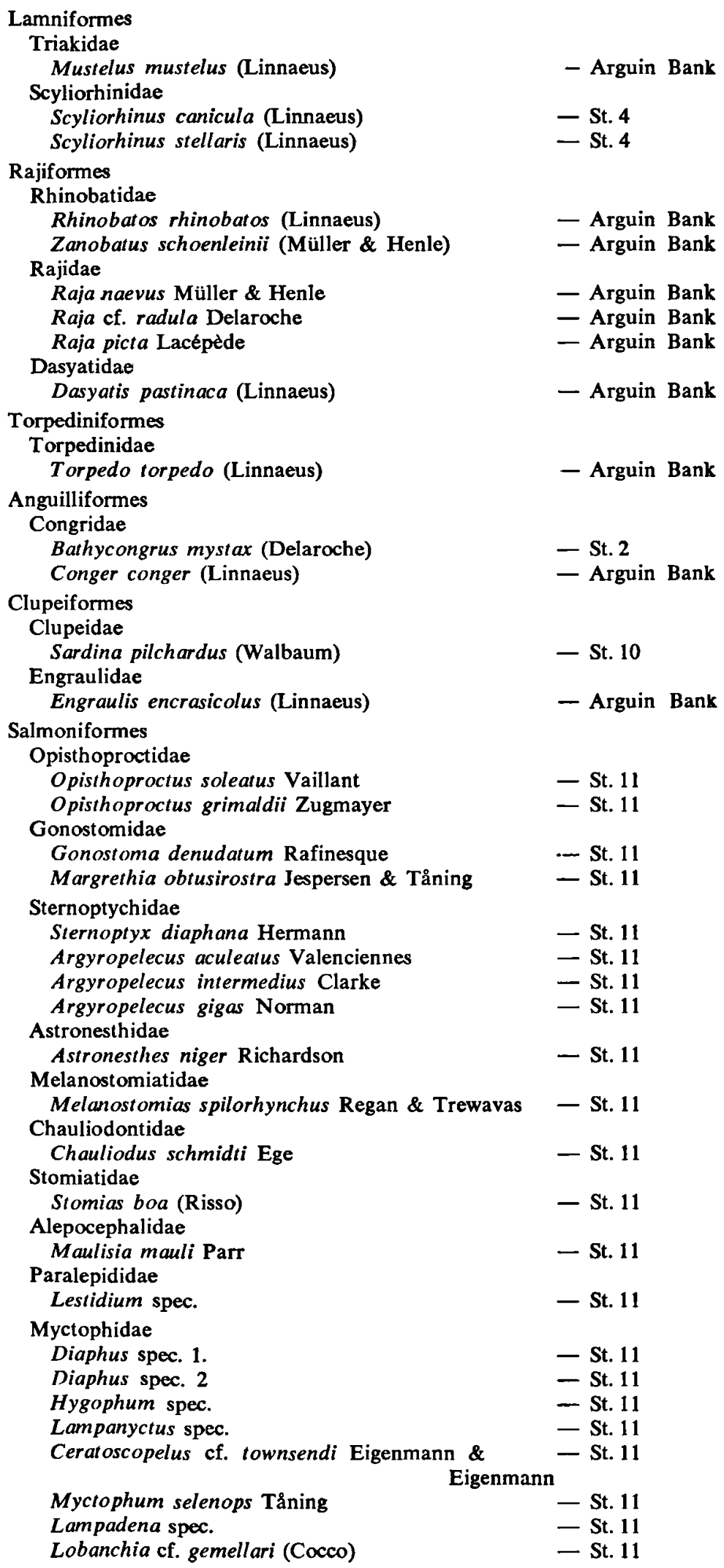




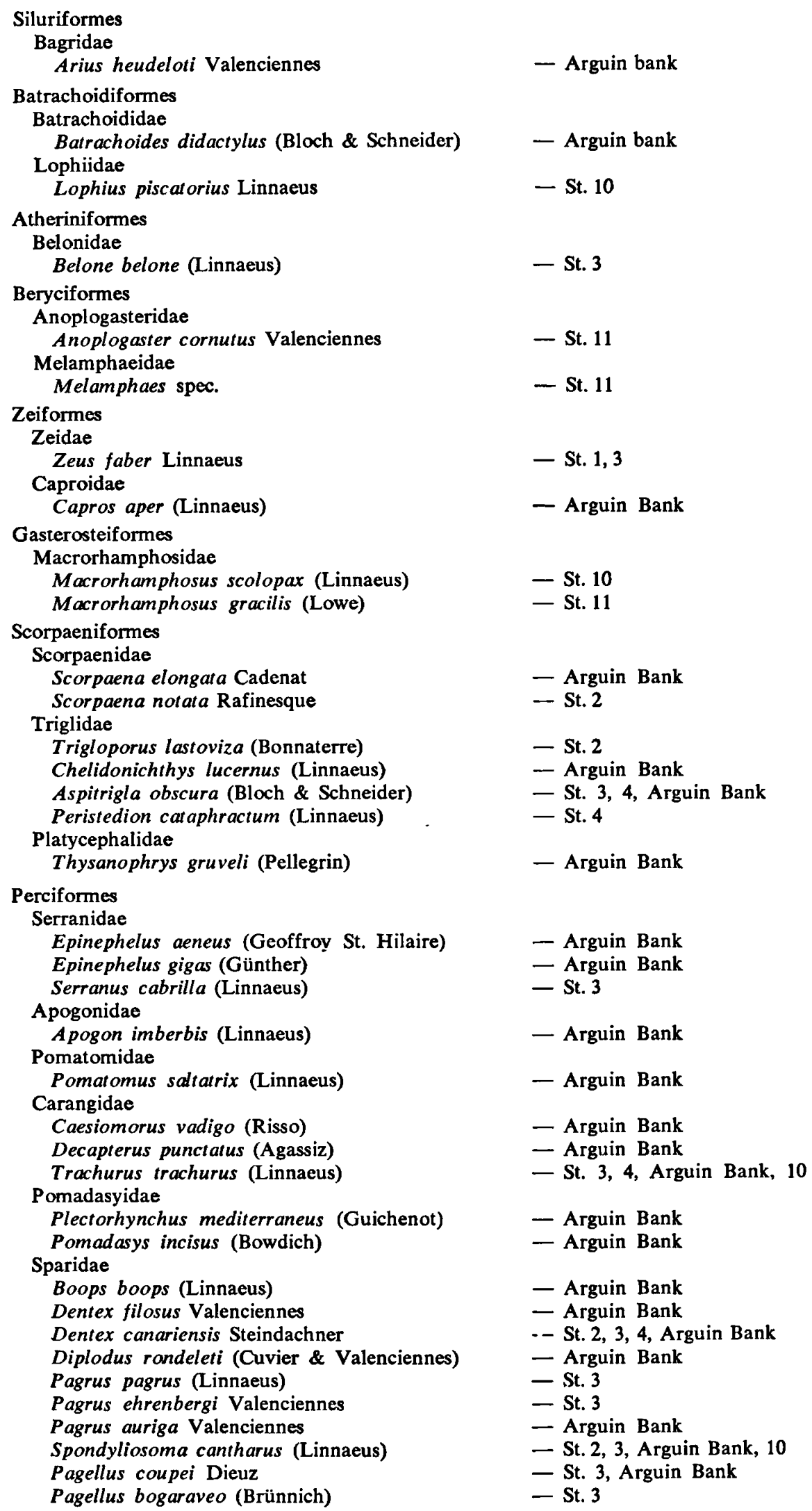



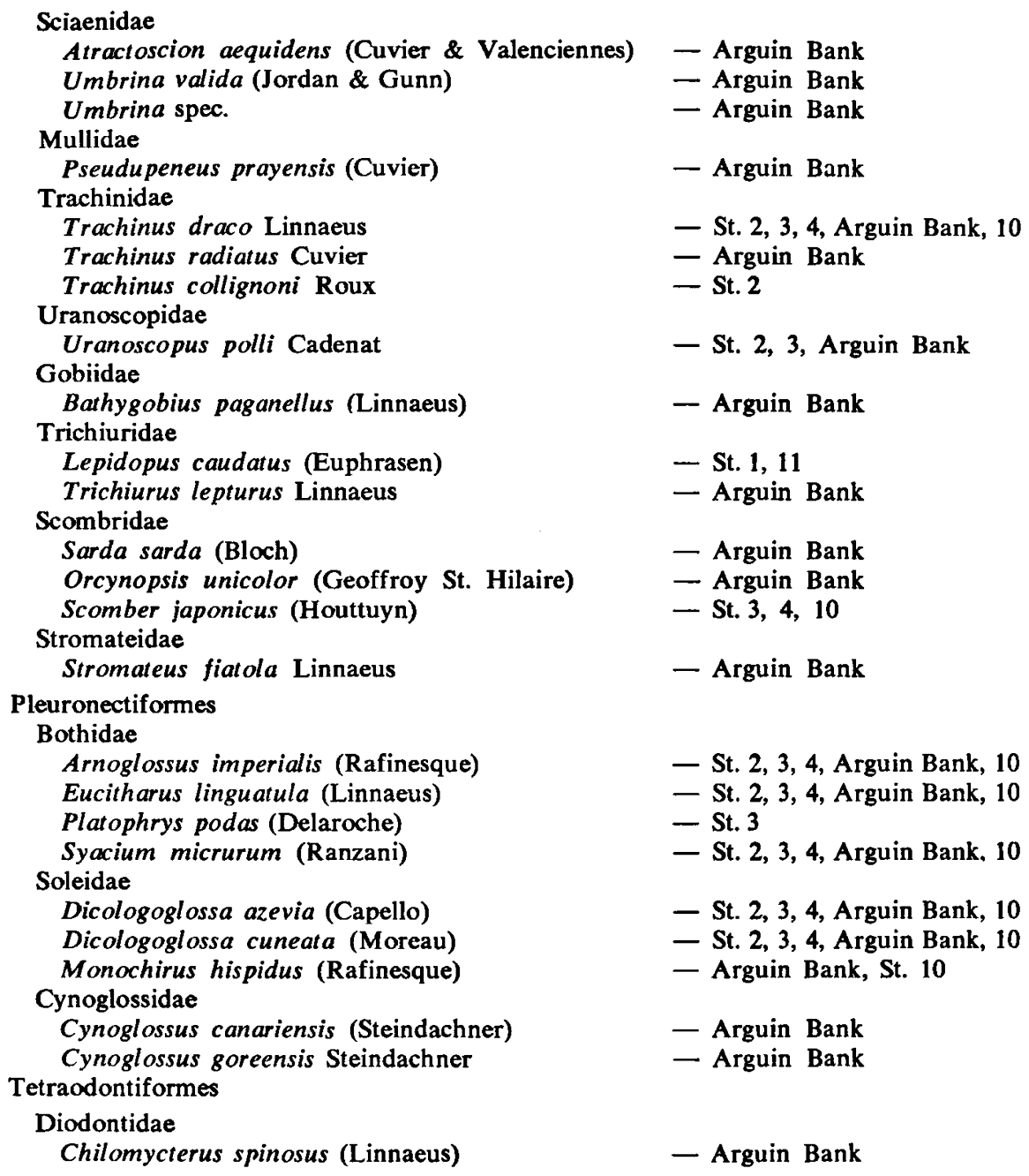

\section{DISCUSSION}

Although there was only little time to spend on this preliminary survey, and though most species listed here are well known from this area, we should like to mention some species which, as far as we know, have hitherto been recorded only farther South.

Atractoscion aequidens (Cuvier \& Valenciennes). Station 5: 2 specimens (ZMA 109.495/496); station 7: 3 specimens (ZMA 109.524); station 8: 4 specimens (ZMA 109.491/494). Northernmost record from $12^{\circ} 54^{\prime} \mathrm{S}, 1^{\circ} 52^{\prime} \mathrm{E}$ (Poll, 1954: 218).

Uranoscopus polli Cadenat. Station 2: 1 specimen (ZMA 109.527); station 10: 1 specimen (ZMA 109.526). Northernmost record from $06^{\circ}$ 07' S, 12 ${ }^{\circ} 12^{\prime}$ E (Poll, 1959: 47).

Platophrys podas (Delaroche). Syn.: Bothus podas. Station 3: 8 specimens (ZMA 109.553).
Although this species is recorded from „Mediterranean and adjacent parts of Atlantic, westwards to the Azores and southwards to Angola" (Norman, 1934: 225), the material from station 3 seems to contradict the existence of the subspecies $P . p$. africanus (Nielsen, 1961). Apart from the subspecies P.p. maderiensis (Lowe, 1834), which is a well distinguishable population off Madeira and the Canary Islands - with 88-91 lateral line scales, and $20-21$ scales in the lateral line curve - Nielsen separated his subspecies africanus from the nominate subspecies as follows: $P$. $p$. podas (Delaroche, 1809), Mediterranean, with 80-84 lateral line scales and 17-20 scales in the lateral line curve. $P$. p. africanus along the West African coast from Cape Blanc to Angola, with 75-82 lateral line scales and 13-17 scales in the lateral line curve. Nielsen obviously did not see Dollfus' paper (1955: 130), because he states "Going 
thoroughly through the literature, I never saw any record of $B$. podas from the coast between Gibraltar and Cape Blanc".

Our eight specimens from $23^{\circ} 09^{\prime} \mathrm{N}, 17^{\circ} 03^{\prime} \mathrm{W}$ were collected just between Gibraltar $\left(36^{\circ} \mathrm{N}, 06^{\circ}\right.$ $\mathrm{W})$ and the type locality of Nielsen's subspecies africanus $\left(09^{\circ} 27^{\prime} \mathrm{N}, 14^{\circ} 48^{\prime} \mathrm{W}\right)$. Our specimens have 76-86 lateral line scales (mean $=79.25$ ), and 16-19 scales in the lateral line curve (mean $=18.25$ ). It seems likely that the number of scales along the lateral line shows gradual (clinical) changes from North to South along the WestAfrican coast, and that $P$. p. africanus should not be regarded a subspecies.
Chilomycterus spinosus (Linnaeus). Station 6:1 specimen (ZMA 109.850). The northernmost record from the literature seems to be $00^{\circ} 33^{\prime} \mathrm{S}$, 08 ${ }^{\circ} 50^{\prime} \mathrm{E}$ (Poll, 1959: 350). Another specimen of this species was caught during the "Guinean Trawling Survey" off Liberia in 1964 (ZMA 108.501).

\section{ACKNOWLEDGEMENT}

The authors are much indebted to the late Mr. Wout E. Pronk of the Technical Research Department of the Fisheries Directorate IJmuiden for his zeal in helping us collecting.

\section{REFERENCES}

AldeberT, Y., 1968. Répartition bathymetrique et géographique des poissons hétérosomes récoltés par la "Thalassa" en 1962 au Rio de Oro et en Mauritanie. CIEM. Symposium sur les ressources vivantes du plateau continental africain du Détroit de Gibraltar au Cap Vert, Section Poissons, 10 : 1-7.

Blache, J., J. Cadenat \& A. Stauch, 1970. Clés de détermination des poissons de mer signalés dans l'Atlantique oriental (entre le $20 \mathrm{e}$ parallèle $\mathrm{N}$. et le $15 \mathrm{e}$ parallèle S.). O.R.S.T.O.M. Faune Tropicale, 18: $1-479$.

Dollfus, R. P., 1955. Première contribution à l'établissement d'un fichier ichthyologique du Maroc Atlantique de Tanger à l'embouchure de l'Oued Dra. Trav. Inst. sci. Chérifien, (Zool.) $6: 1-226$.

FOWLER, H. W., 1936. The marine fishes of West-Africa, based on the collection of the American Museum Congo Expedition, 1909-1915. Bull. Am. Mus. nat. Hist., 70 : 1-2, i-vii, 1-1493.

Lozano Cabo, F., 1968. Relation des espèces de poissons de la région côtière du plateau continental de Maroc, Sahara espagnol et Mauritanie, récueillies durant les campagnes d'investigation faunistique espagnoles. Avec des indications zoogéographiques. CIEM. Symposium sur les ressources vivantes du plateau continental du Détroit de Gibraltar au Cap Vert, Section Poissons, 39 : $1-17$.
Maurin, C., F. Lozano \& M. Bonnet, 1968. Inventaire faunistique des principales espèces ichthyologiques fréquentant les côtes nordouest africaine. Ibid., Section Poissons, $4: 1-13$.

MetzelaAR, J., 1919. Report on the fishes collected by Dr. J. Boeke in the Dutch West Indies 1904-1905. With comparative notes on marine fishes of tropical West Africa, 1-314 (The Hague).

Nielsen, J., 1961. Psettodoidea and Pleuronectoidea (Pisces, Heterosomata). Atlantide Rep., 6 : 101-127.

Norman, J. R., 1934. A systematic monograph of the Flatfishes (Heterosomata), 1. Psettodidae, Bothidae, Pleuronectidae: 1-459 (London).

Poll, M., 1949. Résultats scientifiques des croisières du navire-école belge „Mercator”, 4. Poissons. Mém. Inst. roy. Sci. nat. Belg., (2) 33 : 173-269.

-, 1954. Expédition océanographique belge dans les eaux côtières africaines de l'Atlantique sud (1948-1949). Poissons, 4. Téléostéens Acanthoptérygiens, 1. Mém. Inst. roy. Sci. nat. Belg., 4 (3a) : $1-390$.

-, 1959. Ibid., Poissons, 5: Téléostéens Acanthoptérygiens, 2. Mém. Inst. roy. Sci. nat. Belg., 4 (3b) : $1-417$.

Richards, W. J., 1968. Eastern Atlantic Triglidae (Pisces, Scorpaeniformes). Atlantide Rep., $10: 77-114$. 